\title{
ARE DEICING SALTS NECESSARY TO PROMOTE SCALING IN CONCRETE?
}

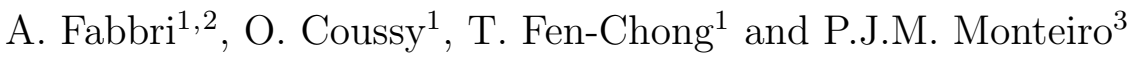

\begin{abstract}
The main purpose of the present study is to investigate the role of the material parameters such as permeability, thermal diffusivity, and pore size distribution on the mechanical behavior of cementitious structures submitted to frost action, such as surface scaling. An experimental device, in which a cement paste specimen is exposed to freezing-thawing cycles under a thermal gradient, has been developed. The experimental results show that under high thermal gradient (up to $1.5^{\circ} \mathrm{C} / \mathrm{mm}$ ), skin damage can occur without a saline layer in contact with the frozen surface. This can be explained and quantified in the framework of poromechanics. The model is based on the coupling between liquid - ice crystal thermodynamic equilibrium, liquid water transport, thermal conduction and elastic properties of the different phases that form the porous material. It eventually predicts that a less permeable sample is more susceptible to be damaged by surface defacement, which explains the observed experimental result.
\end{abstract}

Keywords: Concrete, cryosuction, durability, frost, poromechanics, porous media, thermodynamics, spalling

\section{INTRODUCTION}

When concrete structures are exposed to freezing and thawing cycles, two types of damage can occur: internal frost damage and surface scaling (Pigeon 1984). The former takes place and generates micro-damage within the whole medium. Theoretical study of concrete

\footnotetext{
${ }^{1}$ Université Paris-Est, Institut Navier, LMSGC 2 allée Kepler, 77420 Champs-sur-Marne, France.

${ }^{2}$ new address: Laboratoire de Géologie, UMR 8538 (Ecole Normale Supérieure, CNRS), Paris, France

${ }^{3}$ Department of Civil and Environmental Engineering, University of California at Berkeley, CA, USA.
} 
behavior at low temperature at the material scale started with the work of Powers in 1949 (Powers 1949; Powers and Helmuth 1953). In this seminal work, he attributed the expansion of concrete to the hydraulic pressure originated by the expulsion of liquid water from the freezing pores due to the liquid-to-ice volumetric expansion. Early attempts at evaluating frost performance of concrete were based on the comparison between the tensile stress induced by the hydraulic pore pressure and the tensile strength of the material. However, the picture was not so simple as demonstrated by the fact that expansion is observed in cement paste saturated with benzene, whose density increases with solidification (Beaudoin and MacInnis 1974). Nowadays, it has been recognized that the mechanical response of a saturated or partially saturated porous material at freezing temperatures is the result of the volumetric increase of water during its solidification, the transport of unfrozen liquid water through the porous network and the thermo-mechanical properties of all the phases of the composite material. Physico-mechanics based models have been developed to capture and quantify all these phenomena both at the pore scale (Vignes and Dijkema 1974; Coussy and Fen-Chong 2005) and at the material scale (Coussy 2005; Zuber and Marchand 2004; Bazant et al. 1988).

On the other hand, scaling is the result of a local flaking or peeling of the concrete surface. Generally, it starts as localized small patches which later on often merge and extend to larger areas. Moderate and severe scaling expose the coarse aggregate from the concrete surface and may involve losses up to 3 to $10 \mathrm{~mm}$ of the surface, which is harmful as it reduces the cover of the steel reinforcement. The discovery of scaling in the early 1950s prompted a series of experimental studies (Verbeck and Klieger 1957; Sellevold and Farstad 1991; Pigeon et al. 1996; Valenza and Scherer 2005; Penttala 2006). The results indicate that scaling is largely enhanced by the presence of deicing salts and that any surface desaturation prevents the specimen from scaling. Despite the numerous studies, the mechanisms responsible for surface damage, and especially the action of deicing salts, have not been clearly identified. Indeed, while according to many studies scaling only happens when a saline layer is in contact 
with the surface submitted to frost action (Sellevold and Farstad 1991; Valenza and Scherer 2005), in others (Verbeck and Klieger 1957; Penttala 2006), scaling can happen even when the saline surface is not present, albeit not often.

This work investigates the role of the material parameters such as permeability, thermal diffusivity, pore radii distribution and connectivity (which determines the ice content as a function of temperature at the scale of the representative volume element) on the mechanical behavior of cementitious materials submitted to frost action. Let us emphasize that its goal is not to predict in-situ scaling but to understand how these material parameters may influence the mechanical response of a cementitious structure submitted to frost action and to determine if the action of deicing salts is the only phenomenon that produces surface deterioration.

It is first shown experimentally that scaling can occur without the presence of deicing salts. This experimental result is then explained by a poromechanical-based approach at a scale where the importance of the permeability and the amount of ice formed on frost durability is indubitable.

\section{EXPERIMENTAL EVIDENCE OF SCALING WITHOUT SALTS}

Samples of hardened cement paste, with 0.4 water-cement ratio $(\mathrm{W} / \mathrm{C})$ by mass, were prepared in a 5-liter mortar mixer, and cast in $150 \mathrm{~mm}$ high cubic moulds. Ordinary Portland Cement similar to ASTM Type I and distilled water were used. One day after casting, the specimens were removed from their mould and stored in moist condition (relative humidity $=95 \% \pm 5 \%$ ) for 6 months, when they were cored and cut into $20 \mathrm{~mm}$-thick slices with $40 \mathrm{~mm}$ diameter and remained in water until tested. Some specimens (index $d$ ) were dried in an oven at $55^{\circ} \mathrm{C}$ then saturated with degassed distilled water at $3 \mathrm{kPa}$ air pressure before tested.

The tested sample was inserted between two hollow pistons each filled with a fluid from a cryostat. Their temperature was controlled by a PT100 sensor. The piston in contact with the bottom side of the specimen was held at a constant temperature of $10^{\circ} \mathrm{C}$. The other one was subjected to 56 cycles ranging from $0.1^{\circ} \mathrm{C} \pm 0.1^{\circ} \mathrm{C}$ to $-20^{\circ} \mathrm{C} \pm 0.1^{\circ} \mathrm{C}$. As sketched 
in figure 1 , the temperature rate was $10^{\circ} \mathrm{C} /$ hour. Freezing was stopped at $-20^{\circ} \mathrm{C}$ and the sample was thawed to $0.1^{\circ} \mathrm{C}$. At the end of freezing, the temperature was held constant for one hour and at the end of thawing the temperature was held constant for two hours. Let us recall that the purpose of this paper is not to simulate and/or to predict in-situ frost scaling, but to investigate if scaling can occur without deicing salt. In this context, in order to enhance the effect of structural gradients, a large thermal gradient through the sample was intentionally imposed.

The thermal insulation of the lateral surfaces of the specimen was achieved by an expanded polystyrene ring. In order to avoid surface desaturation during freezing-thawing cycles, each specimen was wrapped by a moisture resistant Parafilm sheet. Thus, the surface submitted to frost action is not in contact with a frost layer (i.e. a water or brine layer). A picture of the experimental device is reported in figure 2 .

After each fourteen cycles, the specimen was weighed in order to verify that no water supply nor evaporation have occurred during the test. Then, the Parafilm sheet was removed and scales were collected, dried at $55^{\circ} \mathrm{C}$ during 4 days and weighed.

Table 1 shows the evolution of the mass of scales collected per unit of surface. As it can be seen, no scaling occurs on previously dried P4-E-3d and P4-E-4d samples. Indeed, these two specimens were totally disintegrated by internal cracking during the first fourteen cycles (see figure 3). On the other hand, a significant scaling occurred on P4-E-1 and P4-E-2 samples (figure 4). This result clearly indicates that scaling can occur without a frozen brine layer in contact with the surface submitted to frost action. The lack of scaling without free liquid on the surface commonly observed (Verbeck and Klieger 1957) may be due to a surface desaturation during the test, which is not possible in this study thanks to the presence of the parafilm sheet.

In all cases, the solicitation was the same and the differences only relied on the cure of the specimen (pre-dried or not). The next step is then to identify the material characteristics which are significantly changed by a drying-resaturation process and to investigate their 
actions on frost-thaw behavior.

\section{CHARACTERIZATION OF MATERIAL PROPERTIES}

It is established that drying strongly affects the intrinsic permeability $\left(\kappa_{0}\right)$ of concrete (Hearn and Morley 1997) and the amount of ice formed during freezing (Fabbri et al. 2006; Kaufmann 1999). The permeability of the pre-dried sample is evaluated through the KatzThompson-Garboczi relation based on Mercury Injection Experiments (Katz and Thompson 1986; Garboczi and Bentz 1996) (see appendix 1). This is not possible for virgin specimens because MIP measurements can only be made on dried samples. In consequence, their permeability is estimated from the predried one according to the study performed by (Hearn and Morley 1997), where 2 orders of magnitude are observed between the permeability of a virgin and a predried sample. This can be explained by the formation of micro-cracks produced by oven-drying that noticeably increases connectivity and size of capillary pores and thus makes the liquid transport easier (Shafiq and Cabrera 2004). Results lead to $\kappa_{0}=4.3 \times 10^{-20} \mathrm{~m}^{2}$ for pre-dried samples and $\kappa_{0}=4.3 \times 10^{-22} \mathrm{~m}^{2}$ for virgin ones. The porosity $\left(\phi_{0}\right)$ was determined through the mass loss between the saturated $\left(m_{\text {sat }}\right)$ and the oven-dried at $55^{\circ} \mathrm{C}\left(m_{d r y}\right)$ states: $\phi_{0}=\left(m_{\text {sat }}-m_{d r y}\right) / \rho_{l}^{0} / V$ where $\rho_{l}^{0}$ is the mass density of liquid water and $V$ is the sample volume. This leads to $\phi_{0}=0.29$ for pre-dried samples and $\phi_{0}=0.28$ for virgin ones. The dependency of the volumetric ratio of unfrozen water $\left(S_{l}\right)$ as a function of temperature $(\theta)$ was determined by a capacitive sensor apparatus developed at the Navier Institute. The full description of the experimental set-up and its calibration are reported in (Fen-Chong et al. 2004) and (Fabbri et al. 2006). As shown in figures 5 and 6 , in the $\left[-30^{\circ} \mathrm{C} ; 0^{\circ} \mathrm{C}\right]$ temperature range, both $S_{l}(\theta)$ curves varies linearly, except for two particular temperatures on cooling, around $-5^{\circ} \mathrm{C}$ and $-20^{\circ} \mathrm{C}$, and one, around $-5^{\circ} \mathrm{C}$ on heating, where the slope changes significantly. However, the amount of ice formed in pre-dried hardened cement pastes appears to be significantly higher than in virgin ones.

Assuming that the in-pore ice formation results from the propagation of ice crystals through the connected porous network (Scherer 1993), the mechanical (Young-Laplace's 
law) and chemical equilibria between ice crystal and surrounding water provide a relation between the water to ice transition temperature $\theta_{l \rightarrow c}$ and the ice crystal mean curvature c* (Brun et al. 1977):

$$
\theta_{l \rightarrow c} \approx-\frac{\gamma c^{*}}{\Sigma_{f}}
$$

where $\gamma$ is the liquid-ice surface tension while $\Sigma_{f}$ stands for the entropy of fusion.

As $c^{*}$ increases with decreasing pore radius in which the water is confined, pores with larger radius will freeze at higher temperature. From studies made with low temperature calorimetry (Sellevold and Bager 1980) and nuclear magnetic resonance (Jehng et al. 1996), a maximum of three distinct types of pores, associated with the three freezing peaks, was observed in a freezing test: large capillaries $\left(r_{c}>50 \mathrm{~nm}, \theta_{l \rightarrow c}>-1^{\circ} \mathrm{C}\right)$, small capillaries $\left(r_{c}=2 \mathrm{~nm}\right.$ to $\left.5 \mathrm{~nm}, \theta_{l \rightarrow c} \simeq-25^{\circ} \mathrm{C}\right)$ and open gel pores $\left(r_{c}<1 \mathrm{~nm}, \theta_{l \rightarrow c}<-40^{\circ} \mathrm{C}\right)$. In our experiment, the lowest temperature is $-30^{\circ} \mathrm{C}$, so only the water confined in large and small capillaries can freeze. This is why only two freezing peaks are presented in the figures 5 and 6. As a consequence, the larger amount of ice formed in P4-E-3d and 4d cement pastes samples can be explained by the damage caused by micro-cracks produced by oven-drying that reduces the proportion of open gel pores and raises the proportion of small and large capillary pores.

The characteristics of the hardened cement paste samples are summarized on table 2 .

At this point, strong differences on permeability and amount of ice formed between the two kinds of specimens have been quantified. Now, in order to evaluate and predict the consequence of these differences on the frost-thaw behavior, theoretical modelling is necessary.

\section{POROMECHANICS OF A PARTIALLY FROZEN CONTINUUM UNDER A THERMAL GRADIENT}

The poromechanical model built in this study assumes that the thermodynamic equilibrium between water in liquid form (index $l$ ) and its ice crystal (index $c$ ) is reached at any 
time. This requires the equality of their specific chemical potentials:

$$
\mu_{l}=\mu_{c}
$$

The natural arguments of the chemical potential $\mu_{j}$ per mass unit of phase $j=l$ or $c$ are the absolute temperature $T$ and the current pressure $p_{J}$. The differentiation of $(2)$ and standard thermodynamics methods give:

$$
\frac{1}{\rho_{l}} \mathrm{~d} p_{l}-s_{l} \mathrm{~d} T=\frac{1}{\rho_{c}} \mathrm{~d} p_{c}-s_{c} \mathrm{~d} T
$$

where $\rho_{j}$ and $s_{j}$ are the density and entropy per unit of mass of the phase $j$.

Hereafter the atmospheric pressure (conveniently set equal to zero in all that follows) and the corresponding freezing point $T_{0}=273.15 \mathrm{~K}$ are adopted as the common reference for pressure and temperature. In this study, small transformations and small displacements are considered. Let $K_{j}$ and $\alpha_{j}$ be the bulk modulus and the volumetric thermal coefficient of the phase $j$. As shown by (Lide 2001), the variation of $K_{j}$ and $\alpha_{c}$ with temperature can be neglected while the variation of $\alpha_{l}$ one can not. The linear form $\alpha_{l}=\alpha_{l}^{0}+\Delta \alpha_{l} \theta$ for the thermal dilatation of supercooled water obtained from the linearization of (Speedy 1987) data in the $\left[-20^{\circ} \mathrm{C}, 0^{\circ} \mathrm{C}\right]$ temperature range is adopted. Then, under the hypothesis of small density variations, the density of liquid water and ice crystal are:

$$
\begin{aligned}
& \rho_{l}=\rho_{l}^{0}\left(1+\frac{p_{l}}{K_{l}}-\left(\alpha_{l}^{0}+\Delta \alpha_{l} \frac{\left(T-T_{0}\right)}{2}\right)\left(T-T_{0}\right)\right) \\
& \rho_{c}=\rho_{c}^{0}\left(1+\frac{p_{c}}{K_{c}}-\alpha_{c}^{0}\left(T-T_{0}\right)\right)
\end{aligned}
$$

where $\rho_{l}^{0}, \rho_{c}^{0}, \alpha_{l}^{0}$ and $\alpha_{c}^{0}$ stand for the reference state value of $\rho_{l}, \rho_{c}, \alpha_{l}$ and $\alpha_{c}$.

Assuming that the entropy $s_{j}$ of the phase $j$ per unit of porous media volume does not depend on pressure, standard thermodynamics leads to: 


$$
s_{j}=s_{j}^{0}+c_{p, j}^{0} \ln \frac{T}{T_{0}}
$$

where $s_{j}^{0}$ and $c_{p, j}^{0}$ are the bulk specific entropy and the specific heat capacity, both constant and associated with the reference state while $T_{0}$ is the freezing temperature of bulk liquid water.

Let us define the capillary pressure as $p_{c a p}=p_{c}-p_{l}$. The integration of $(3)$ from the reference state, while using (4-5), the hypothesis of small density variations and neglecting second order terms, but taking into account variations of temperature from 253.15 to 273.15 K leads to (see (Fabbri 2006) for more details):

$$
p_{\text {cap }}=\Sigma_{f}\left(T_{0}-T\right)-\mathcal{C}_{f} \frac{\left(T-T_{0}\right)^{2}}{2 T_{0}}
$$

where $\Sigma_{f}=\rho_{c}^{0}\left(s_{l}^{0}-s_{c}^{0}\right)$ and $\mathcal{C}_{f}=\rho_{c}^{0}\left(c_{p, l}^{0}-c_{p, c}^{0}\right)$ are respectively the entropy of fusion and the heat capacity difference between water and ice per unit of volume.

\section{Unsaturated poroelasticity}

Let us consider the initial state to be $[\sigma](\underline{x}, 0)=0,[\varepsilon](\underline{x}, 0)=0, p_{l}(\underline{x}, 0)=p_{c}(\underline{x}, 0)=0$, $T(\underline{x}, 0)=T_{0}, \Sigma(\underline{x}, 0)=\Sigma_{0}$ and $\varphi_{l}(\underline{x}, 0)=\varphi_{c}(\underline{x}, 0)=0$ where $[\sigma],[\varepsilon]$ and $\Sigma$ are respectively the stress tensor, the strain tensor and the entropy of the empty porous medium (solid matrix and empty porous network) while $\varphi_{j}$ is the deformation of the porosity occupied by phase $j$. The relationship between the related porosity of the phase $j$ (noted $\phi_{j}$ ), the saturation ratio of the phase $j$ and $\varphi_{j}$ is given by (Coussy 2005):

$$
\phi_{j}=\varphi_{j}+\phi_{0} S_{j}
$$

The lowest temperature reached in the scaling experiments is $-20^{\circ} \mathrm{C}$. As indicated in figures 5-6 and in table 2 , less than $60 \%$ of water is solidified in the tested samples. The interfacial liquid-ice energy remains small enough to be negligible in the expression of the pressure 
actually transmitted to the solid matrix by the crystal and liquid phases(Fabbri 2006). In this case the complete set of constitutive equations of the empty porous medium (Coussy 2004; Coussy 2005) are then found to simplify to (Fabbri 2006; Coussy and Monteiro 2007):

$$
\begin{aligned}
{[\sigma] } & =\left(K-\frac{2}{3} G\right) \epsilon[1]+2 G[\varepsilon]-\left(\sum_{j=l, c}\left(b_{j} p_{j}\right)+\alpha K\left(T-T_{0}\right)\right)[1] \\
\varphi_{j} & =b_{j} \epsilon+\frac{1}{N_{j l}} p_{l}+\frac{1}{N_{j c}} p_{c}-\alpha_{\phi j}\left(T-T_{0}\right) ; \quad j=l \text { or } c \\
\Sigma-\Sigma_{0} & =\alpha K \epsilon-\sum_{j=l, c}\left(\alpha_{\phi j} \mathrm{~d} p_{j}\right)+C_{m} \ln \left(\frac{T}{T_{0}}\right)
\end{aligned}
$$

where $\epsilon=\operatorname{tr}([\varepsilon])$ is the volumetric dilatation; $K, G, C_{m}$ and $\alpha$ are respectively the bulk modulus, the shear modulus, the heat capacity, and the thermal volumetric dilatation coefficient of the empty porous medium; $b_{j}$ and $N_{i j}$ are the generalized Biot coefficients and the generalized Biot coupling moduli while $\alpha_{\phi j}$ is the coefficient related to the thermal dilatation of the pore volume occupied by phase $j$. They depend upon the elastic properties of the solid matrix and the saturations of liquid and ice according to:

$$
\frac{1}{N_{j j}}+\frac{1}{N_{l c}}=\frac{b_{j}-\phi_{0} S_{j}}{K_{m}} ; b_{c}+b_{l}=1-\frac{K}{K_{m}} \text { and } \alpha_{\phi_{j}}=\alpha\left(b_{j}-\phi_{0} S_{j}\right) \text { where } j=l \text { or } c
$$

with $K_{m}$ the bulk modulus of the solid matrix.

The generalized Biot coefficients $b j$ can be estimated from the Biot coefficient $b=$ $1-K / K_{m}$ by use of the so-called iso-deformation hypothesis expressing that there is no morphological difference between the forming ice crystal and the liquid water domains (they are both bounded by pores having the same shape). This yields (Chateau and Dormieux 2002):

$$
b_{j}=S_{j} b ; j=c, l
$$


Let us assume an one-dimensional problem without any external mechanical load. The momentum balance $\left(\operatorname{div}\left(\sigma_{i j}\right)=0\right)$ and the geometric compatibility of the strain tensor $\left([\varepsilon]=[\operatorname{grad}(\underline{u})]+[\operatorname{grad}(\underline{u})]^{\mathrm{T}}\right)$, where $\underline{u}$ is the displacement vector, require $\sigma_{i j, i \neq j}$ and $\sigma_{x x}$ to be null everywhere, while only the $\varepsilon_{x x}$ term of the strain tensor $\varepsilon_{i j}$ is non zero:

$$
\sigma_{i j, i \neq j}=\sigma_{x x}=0 ; \varepsilon_{x x}=\frac{\partial u_{x}}{\partial x} ; \varepsilon_{i j}=0 \text { for } i j \neq x x
$$

Then, the substitution (6), (9) and (11) in the constitutive equation (8a) allows for the computation of volumetric strain:

$$
\epsilon=\frac{b p_{l}+b_{c}\left(\mathcal{C}_{f}\left(T-T_{0}-T \ln \frac{T}{T_{0}}\right)-\Sigma_{f}\left(T-T_{0}\right)\right)+\alpha K\left(T-T_{0}\right)}{K+4 G / 3}
$$

Due to the temperature gradient across the specimen, the pressure field is not uniform and a liquid flow is created. Assuming that it is governed by Darcy's law, we write:

$$
\underline{\omega}=-\rho_{l}^{0} \frac{\kappa\left(S_{l}\right)}{\eta_{l}\left(T, p_{l}\right)} \underline{\operatorname{grad}}\left(p_{l}\right)
$$

where $\underline{\omega}$ is the relative flow vector of mass fluid, while $\kappa\left(S_{l}\right)$ and $\eta_{l}\left(T, p_{l}\right)$ are respectively the permeability of the porous medium and the viscosity of liquid water. The expression of the permeability has been extensively studied in case of drying, as reviewed in (Baroghel-Bouny et al. 2007). In case of freezing/thawing, the NMR-based paper (Kleinberg and Griffin 2005) concludes that ice forms from the pore centres. Since no liquid flow can occur through ice crystal, such a morphology can be represented by the usual self-consistent differential scheme yielding (Dormieux and Bourgeois 2003):

$$
\kappa=\kappa_{0}\left(S_{l}\right)^{3 / 2}
$$

where $\kappa_{0}$ is the constant intrinsic permeability, while the term $\left(S_{l}\right)^{3 / 2}$ stands for the relative permeability accounting for the change in porous volume due to the solidification of liquid 
water.

Because the flow of ice is significantly slower than the usual cooling timescales, it will be neglected. In this case, the overall mass conservation of water $\left(m=m_{l}+m_{c}\right)$, in both liquid and crystal forms, requires:

$$
\frac{\partial m}{\partial t}=-\operatorname{div}(\underline{\omega})
$$

Using (7), the global mass of liquid and crystal per unit of porous medium volume can be expressed as:

$$
m_{j}=\rho_{j} \phi_{j}=\rho_{j}\left(\phi_{0} S_{j}+\varphi_{j}\right) ; j=l \text { or } c
$$

Finally, due to the small density difference between liquid and crystal phases of water $\left(\left|\rho_{c}^{0} / \rho_{l}^{0}-1\right| \approx 0.09 \ll 1\right)$, the combination of (4), (8b), (9-10), (12), (13), (15) and (16) under the assumption of small perturbation allows us to express the overall mass conservation according to the only unknown variables $p_{l}$ and $T$ :

$$
\operatorname{div}\left[\rho_{l}^{0} \frac{\kappa\left(S_{l}\right)}{\eta_{l}\left(T, p_{l}\right)} \underline{\operatorname{grad}}\left(p_{l}\right)\right]=\frac{\partial}{\partial t}\left(A p_{l}\right)+\dot{r}_{\Delta \rho}+\dot{r}_{T}+\dot{r}_{c r y o}
$$

with:

$$
\begin{aligned}
A & =\phi_{0} S_{c}\left(\frac{1}{K_{c}^{0}}-\frac{1}{K_{l}^{0}}\right)+\frac{b^{2}}{K+4 G / 3}+\frac{b-\phi_{0}}{K_{m}}+\frac{\phi_{0}}{K_{l}} \\
\dot{r}_{\Delta \rho} & =\phi_{0}\left(\frac{\rho_{c}^{0}}{\rho_{l}^{0}}-1\right) \frac{\partial S_{c}}{\partial t} \\
\dot{r}_{T} & =\left(\phi_{0}\left[\alpha-\alpha_{l}^{*}+S_{c}\left(\alpha_{l}^{*}-\alpha_{c}^{*}\right)\right]-\alpha b \frac{4 G / 3}{K+4 G / 3}\right) \frac{\partial T}{\partial t} \\
\dot{r}_{p_{c a p}} & =\left(\phi_{0} S_{c}\left(\frac{1}{K_{m}}-\frac{1}{K_{c}^{0}}\right)-b_{c}\left(\frac{b}{K+4 G / 3}+\frac{1}{K_{m}}\right)\right) \frac{\partial p_{c a p}}{\partial t}
\end{aligned}
$$

The source term of liquid pressure in (17) is made of three distinct contributions. The first contribution, $\dot{r}_{\Delta \rho}$, is a positive term originating from the expansion occurred when liquid water freezes. It is the only contribution considered in Power's hydraulic theory (Powers 1949). The term $\dot{r}_{T}$ accounts for the pressure in the liquid caused by the relative 
thermal contraction between the solid matrix and the in-pore constituent. And finally, $\dot{r}_{p_{c a p}}$ accounts for the pressure in the liquid caused by flows at the microscopic scale. The physical understanding of the driving force of these flows, which drives liquid water to the already frozen sites in order to meet the liquid-crystal equilibrium condition (6), was previously discussed in (Vignes and Dijkema 1974; Vignes-Adler 1976).

\section{Equation of heat transfer}

The second law of thermodynamic applied to the porous medium for a reversible evolution leads to:

$$
T\left(\frac{\partial \Sigma_{s}}{\partial t}+m_{l} \frac{\partial s_{l}}{\partial t}+m_{c} \frac{\partial s_{c}}{\partial t}\right)=T\left(s_{l}-s_{c}\right) \frac{\partial m_{c}}{\partial t}-\operatorname{div}(\underline{q})
$$

where $\Sigma_{s}$ is the entropy of the skeleton, which, in the present study, is assumed to be equal

to the entropy of the empty porous medium $\Sigma$. The term $\left(s_{l}-s_{c}\right) \frac{\partial m_{c}}{\partial t}$ is the heat source due to the latent heat of liquid to ice phase change. The heat flow $(\underline{q})$ is unidirectional and the medium is assumed to be isotropic. Applying Fourier's Law:

$$
\underline{q}=-\lambda\left(S_{l}\right) \frac{\partial T}{\partial x} \underline{x}
$$

where $\lambda\left(S_{l}\right)$ is the isotropic thermal conductivity. Assuming that ice crystal is growing from the center of the pore in which it is confined, $\lambda$ can be estimated using the $(n+1)$-phases multi-scale scheme developed by (Hervé 2002) for isotropic composite media. Thus:

$$
\begin{array}{r}
\lambda\left(S_{l}, T\right)=\lambda_{m}(T)\left(1+\frac{\phi_{0}}{\frac{1-\phi_{0}}{3}+\frac{\lambda_{m}(T)}{\lambda_{i}\left(S_{l}, T\right)-\lambda_{m}(T)}}\right) \\
\text { with } \lambda_{i}\left(S_{l}, T\right)=\lambda_{l}(T)\left(1+\frac{1-S_{l}}{\frac{S_{l}}{3}+\frac{\lambda_{l}(T)}{\lambda_{c}(T)-\lambda_{l}(T)}}\right)
\end{array}
$$

where $\lambda_{s}, \lambda_{l}$ and $\lambda_{c}$ are the isotropic thermal conductivity of matrix, liquid water and ice crystal respectively. 
Moreover, assuming that the entropy of the skeleton $\Sigma_{s}$ does not depend on pressure and strain, and under the small transformation hypothesis, (8c) and (16) gives the following approximation for $\frac{\partial \Sigma_{s}}{\partial t}$ and $\frac{\partial m_{c}}{\partial t}$ :

$$
\begin{aligned}
\frac{\partial \Sigma_{s}}{\partial t} & =C_{m} \frac{\partial T}{\partial t} \\
\frac{\partial m_{c}}{\partial t} & =-\rho_{c}^{0} \phi_{0} \frac{\partial S_{l}}{\partial t}
\end{aligned}
$$

Let $C_{p l}=\rho_{l}^{0} c_{p, l}^{0}$ be the heat capacity of water per volume unit. The substitution of (20) and (22) in (19) leads to:

$$
\operatorname{div}\left(\lambda \frac{\partial T}{\partial x} \underline{x}\right)=\frac{\partial T}{\partial t}\left[\left(C_{m}+\phi_{0}\left(C_{p l}-S_{c} \mathcal{C}_{f}\right)\right)-T \phi_{0}\left(\Sigma_{f}+\mathcal{C}_{f} \ln \frac{T}{T_{0}}\right) \frac{\partial S_{c}}{\partial T}\right]
$$

where $-T \phi_{0}\left(\Sigma_{f}+\mathcal{C}_{f} \ln \frac{T}{T_{0}}\right) \frac{\partial S_{c}}{\partial T}$ stands for the latent heat of solidification (resp. fusion) of water and $\mathcal{C}_{m}+\phi_{0}\left(\mathcal{C}_{p l}-S_{c} \mathcal{C}_{f}\right)$ for the average heat capacity of the porous medium.

\section{NUMERICAL APPLICATION}

The specimen is modelled as a one-dimensional structure made up of an isotropic medium, of length $L$ and lateral surface $S$, ideally insulated on its lateral surfaces. The Cartesian coordinate system $(O, \underline{x})$ is used, with $O$ the node of the surface which is submitted to frost action and $\underline{x}$ following the symmetry axis from the top to the bottom of the specimen. In this case, at the macroscopic scale the flow of heat and liquid only occurs in the $\underline{x}$ direction. As a consequence, the elementary volume is given by $\mathrm{d} \Omega=S \mathrm{~d} x$.

At the initial condition, the temperature of the sample is $T(x, t=0)=T_{0}=273.15 \mathrm{~K}$ with no water overpressure $\left(p_{l}(x, t=0)=0 \mathrm{MPa}\right)$ where $T$ stands for absolute temperature in Kelvin $\left(T[\mathrm{~K}]=\theta\left[{ }^{\circ} \mathrm{C}\right]+273.15\right)$ and $t$ stands for time in second. At $t=0$, the $x=0$ surface is submitted to a progressive decrease of temperature while the $x=L$ surface is 
increased to $T_{b}=283.15 \mathrm{~K}$ :

$$
T(0, t)=T_{s}(t) ; T(L, t)=T_{b}
$$

where $T_{s}(t)$ follows the same time-evolution as the experimental protocol, thus:

$$
\begin{aligned}
& T_{s}(t)=273.15-0.002792 \times t ; t \in[0,7200] \\
& T_{s}(t)=253.15 ; t \in[7200,10800] \\
& T_{s}(t)=253.15+0.002792 \times(t-10800) ; t \in[10800,18000]
\end{aligned}
$$

In this study, no external loading is applied to the structure, which leads to: $[\sigma] \cdot n_{L}=0$ where $n_{L}$ is the outward unit vector of the bottom surface.

The parafilm sheet used to wrap the specimen should not be sufficient to prevent the in-pore water to be expelled from the $x=L$ surface. Consequently, the most realistic liquid pressure boundary condition is:

$$
p_{l}(L, t)=0
$$

Similarly, we may assume that the water which solidifies at the $x=0$ surface is stress-free. Then, the following boundary condition should be taken:

$$
p_{c}(0, t)=0
$$

Alternatively, we may suppose that the water which solidifies at the $x=0$ surface during the first freezing-thawing cycles will play the role of an impermeable frost layer. In this case, no flow $(\underline{\omega})$ through the frozen surface has to be prescribed as boundary condition:

$$
\underline{\omega} \cdot \underline{n_{0}}=0 \text { for } x=0
$$


where $\underline{n_{0}}$ is the outward unit vector perpendicular to the frozen surface.

In the following, the influences of each of (29) and (30) boundary conditions on frost behavior is studied.

At last, the poromechanical-based approach of the problem can be summarized by equations (17-23) where $p_{l}$ and $T$ stand for the main unknown variables. This system is solved using the Newton-Raphson method on a structure discretised according to the finite volume method implicit scheme (Eymard et al. 2000). Mathematical functions used to implement the $S_{l}(\theta)$ experimental curves on the model are reported on appendix 2 and the thermophysical properties values used for the calculation are reported on appendix 3.

The predicted liquid and ice pressure profiles at the end of the cooling stage, for the predried and virgin hardened cement pastes and the (29) and (30) boundary conditions, are on figure 7 .

\section{DISCUSSION}

Whatever the permeability and the boundary condition used, the maximum ice pressure (round to 25.5 $\mathrm{MPa}$ and 41.0 $\mathrm{MPa}$ for the less permeable cement paste and round to 8.5 $\mathrm{MPa}$ and 32.0 $\mathrm{MPa}$ for the more permeable one) is close to or higher than the usual tensile elastic limit of an Ordinary Portland Cement Paste $\left(f_{t}\right)$, which is between 5 and $10 \mathrm{MPa}$ (Taylor, 1997). In case of the $p_{c}(0, t)=0$ boundary condition, the ice pressure peak of the virgin sample is localized at $2.7 \mathrm{~mm}$ beneath the surface, which is in appropriateness with the depth of scaling commonly observed under lower thermal gradients. On the contrary the ice pressure peak of the pre-dried sample is broad and localized in the sample core ( 8 $\mathrm{mm}$ beneath the surface). In case of the $\underline{\omega}(0, t) \cdot \underline{n_{0}}=0$ boundary condition, the profiles shape are also noticeably different. Indeed, for the predried sample, the $p_{c}(x)$ curve is quite homogeneous all over the specimen, while for the virgin specimen a great peak of overpressure is observed near the frozen surface.

However, the knowledge of the ice pressure is not sufficient to estimate the susceptibility of the solid matrix to be crushed. To do so, we rather use the stress in the solid matrix, $\sigma_{m, x x}$. 
As reported in (Chateau and Dormieux 2002), neglecting the influence of the interfacial tension between liquid water and pore walls, it can be linked to the porous medium stress and the pressures of liquid and crystal by the relation $\left(1-\phi_{0}\right) \sigma_{m, x x}=\sigma_{x x}+\phi_{l} p_{l}+\phi_{c} p_{c}$. Then, assuming that the thermophysical properties of the solid matrix are constant over the temperature cycle, $\sigma_{m, x x}$, can be expressed through (6) and (11) as:

$$
\sigma_{m, x x}=\frac{\phi}{1-\phi_{0}} p_{l}-\frac{\phi_{c}}{1-\phi_{0}}\left(\Sigma_{f}\left(T-T_{0}\right)+\mathcal{C}_{f} \frac{\left(T-T_{0}\right)^{2}}{2 T_{0}}\right)
$$

The $\sigma_{m, x x}$ profiles are in figure 8 .

For the pre-dried specimen, whatever the boundary condition used, no peak of tensile stress is predicted near the surface submitted to frost action. Moreover, only the $\underline{\omega}(0, t) \cdot \underline{n_{0}}=$ 0 boundary condition results in a significant risk of frost damage through the structure ( $\sigma_{m, x x}$ is higher than $5 \mathrm{MPa}$ until $8 \mathrm{~mm}$ depth). Then, the hypothesis of the existence of an impermeable frozen film on the skin surface leads to a better suitability between experimental results and theoretical calculations. For virgin samples, both boundary conditions give a matrix stress close to or higher than $f_{t}$ only within the first millimeters depth (until $3 \mathrm{~mm}$ ). This fact, enhanced by a poor surface quality of the material and an increasing number of freezing-thawing cycles, can explain the observed damage on the skin surface.

The explanation of the behavior difference between the two types of samples is pointed up by the $p_{l}(x)$ profiles. Indeed, liquid over-pressure appears to be easily dissipated through the porous network for a pre-dried sample than in a virgin one, where liquid over-pressurization peaks are observed. This is why, while the amount of ice formed in pre-dried samples is significantly higher than in virgin ones $\left(\right.$ at $-20^{\circ} \mathrm{C}, S_{c}$ is about 0.1 for a virgin sample and $S_{c}$ is about 0.5 for a predried one), the maximum ice pressure of the virgin sample is higher: a higher permeability allows a more efficient in-pore overpressure relaxation.

Actually, in case of too low permeable cement pastes, the liquid flow expulsion is not important enough to relax the whole local overpressure caused by thermal stresses, cryogenic aspiration and liquid-ice difference of density. Thus, this pore overpressure, which produces 
a local tensile stress on the matrix, remains localized near the $x=0$ surface where the ice formation is the most important. This fact is illustrated on figure 9 where a decrease of the maximum tensile stress on the matrix is predicted during the 1 hour temperature step at $-20^{\circ} \mathrm{C}$. This decrease is clearly due to the pore relaxation due to slow water transport from over-pressurized zone to the bottom and/or the top of the specimen.

This explanation leads to the conclusion that scaling is enhanced by the inability of the frozen porous network to relax local overpressure from the skin surface. This is consistent with the experimental data from (Baroghel-Bouny et al. 2002) where a concrete with a compressive strength equal to $50 \mathrm{MPa}$ exhibits a better frost durability than a less permeable but higher resistance concrete (with a compressive strength equal to $75 \mathrm{MPa}$ ). In addition, the common lack of efficiency of air-entraining agent in a high resistant concrete (LCPC 2003) can then be attributed to the difficulty of the liquid water to migrate from the porous network to air voids in case of too low permeable material.

To sum up, similarly to the experimental observations, the poromechanical model rather predicts an internal frost damage for pre-dried samples and scaling for virgin samples even though they are submitted to the same solicitation. The observed difference between the two mechanisms seems to be caused by the contrast between permeability and amount of ice formed. Indeed, in the case of initial and boundary conditions which force the top surface temperature to be lower than the bottom one, water will first freeze near the skin surface. If the amount of ice formed is high enough and the permeability is too small to relax pore overpressure, scaling will occur. Thus, it appears to be an "internal frost"-like damage localized at the $x=0$ surface.

According to a recent work performed by (Valenza and Scherer 2005), the increase of scaling due to the presence of salts is a consequence of mechanical interaction between the skin surface and the frozen brine layer. This effect can be taken into account in the present model by changing the boundary conditions at the top surface $(x=0)$. For this case, all components of the strain tensor have to be taken into account. It leads to lengthy three- 
dimensional calculations but the global analysis remains the same.

\section{CONCLUSION}

An experimental device, in which hardened cement paste specimens are submitted to freezing-thawing cycles under a thermal gradient, has been developed. This study reveals occurrence of scaling without a brine layer in contact with the frozen surface. To explain this behavior, a poroelastic model has been worked out. The model predicts that scaling can occur without salts due to the localization of pore overpressure near the skin surface, caused by the low permeability of cementitious material. Finally, a link is established between the frost behavior at the structural scale and the two key material parameters toward frost durability: the permeability and the amount of ice formed as a function of temperature. The permeability influences tensile matrix stress localization while the amount of formed ice rather acts on its global amplitude.

\section{Appendix 1: Permeability of the saturated porous specimen}

The permeability is estimated using a mercury injection experiment and the KatzThompson relation (Katz and Thompson 1986):

$$
\kappa_{0}=\frac{1}{226} l_{c}^{2} \frac{C}{C_{0}}
$$

where $l_{c}$ is the threshold pore diameter in mercury injection experiment (MIE), $C$ the sample conductivity and $C_{0}$ the conductivity of the water in the pore space. The relative conductivity $C / C_{0}$ is also estimated by a mercury injection experiment through relationship reported in (Garboczi and Bentz 1996), based on a 3-D electrical analogy of the cement paste and assuming that the relative conductivity of capillary water is 1 while all the other constituents ones are null:

$$
\frac{C}{C_{0}}=0.001+0.07 \phi_{M I E}^{2}+1.8\left[\left[\phi_{M I E}-\phi_{c}\right]\right]\left(\phi_{M I E}-\phi_{c}\right)^{2}
$$


where $\phi_{M I E}$ is the porosity estimated by a mercury injection experiment while $\phi_{c}$ is the capillary porosity under which the pore space becomes non percolated and is commonly estimated to be approximately 0.18 (Powers 1959; Garboczi and Bentz 1996) [[.]] is the Heaviside function. As reported in (Garboczi and Bentz 1996; Cui and Cahyadi 2001), a good agreement is found between (33) and experimental data for ordinary cement pastes by many authors.

\section{Appendix 2: Fit of the freezing-thawing curves}

The freezing-thawing curves shapes, quite similar to water retention capillary curves ones, are well described by the following "Van-Genuchten"-like (Van Genuchten 1980) sigmoidal functions:

$$
\begin{aligned}
& S_{l, \text { frost }}(\theta)=\sum_{i=1}^{k} \phi_{i}\left[1+n_{i}\left(\frac{\theta}{\theta_{i}^{*}}\right)^{\frac{1}{1-n_{i}}}\right]^{n_{i}}+\left(1-\sum_{i=1}^{k} \phi_{i}\right) \\
& S_{l, \text { thaw }}(\theta)=\phi_{T}+\left(1-\phi_{T}\right)\left(1+n\left(\frac{\theta}{\theta^{*}}\right)^{\frac{1}{1-n}}\right)^{-n}
\end{aligned}
$$

where $S_{l, \text { frost }}(\theta)$ and $S_{l, \text { thaw }}(\theta)$ are the liquid saturation ratio on freezing and thawing, while $k$ stands for the number of freezing peaks, $\theta_{i}^{*}$ for the temperature at which each of them happens, and $\theta^{*}$ for the temperature at which the thawing peak happens. These coefficients can be evaluated from the analysis of the $S_{l}(\theta)$ derivative curve.

Let $\theta_{t h}$ be the temperature at which thawing begins. Because the liquid saturation ratio is a continuous function, $S_{l, \text { frost }}\left(\theta_{t h}\right)$ and $S_{l, t h a w}\left(\theta_{t h}\right)$ are equal. As a consequence, the coefficient $\phi_{T}$ must be:

$$
\phi_{T}=\frac{S_{l, f r o s t}\left(\theta_{t h}\right)-f_{n, \theta^{*}}\left(\theta_{t h}\right)}{1-f_{n, \theta^{*}}\left(\theta_{t h}\right)}
$$

with $f_{n, \theta^{*}}(\theta)=\left(1+n\left(\frac{\theta}{\theta^{*}}\right)^{\frac{1}{1-n}}\right)^{-n}$.

Finally coefficients $\phi_{i}, n_{i}$ and $n$, which are the amplitude and the shape factor of the 
$i^{\text {th }}$ freezing sigmoidal function and the shape factor of the thawing curve, need to be fitted from experimental data. It must also be emphasized that $\theta^{*}$ and $n$ appear to be independent of $\theta_{t h}$ on the two kind of tested cement pastes (virgin or pre-dried). Thus, the fit of only one thawing curve is sufficient to estimate the amount of ice within the porous network whatever the lower temperature reached during the freezing stage. Figures 5 and 6 show the comparison between the experimental data and the fitted curves using equation (34) for different thawing temperatures, keeping $\theta^{*}$ and $n$ constant for each thawing curves.

\section{Appendix 3: Thermophysical properties}

Numerical application is made with $\rho_{l}^{0}=9.97 \times 10^{-3} \mathrm{MN} / \mathrm{m}^{3}, \rho_{c}^{0}=9.17 \times 10^{-3} \mathrm{MN} / \mathrm{m}^{3}$, $\alpha_{l}^{0}=-68.7 \times 10^{-6} \mathrm{~K}^{-1}$ and $\Delta \alpha_{l}=24.732 \times 10^{-6} \mathrm{~K}^{-2}, \alpha_{c}^{0}=160 \times 10^{-6} \mathrm{~K}^{-1}, K_{l}=1970 \mathrm{MPa}$, $K_{c}=4310 \mathrm{MPa}, \lambda_{l}=0.56+0.0017\left(T-T_{0}\right) \mathrm{W} /(\mathrm{mK}), \lambda_{c}=2.15+0.0123\left(T-T_{0}\right) \mathrm{W} /(\mathrm{mK})$ (Lide 2001), $\Sigma_{f}=1.2 \mathrm{MPa} / \mathrm{K}, \mathcal{C}_{f}=2.14 \mathrm{MPa} / \mathrm{K}, \mathcal{C}_{p l}=4 \mathrm{MPa} / \mathrm{K}$ (Brun et al. 1977), $\mathcal{C}_{m}=1,36 \mathrm{MPa} / \mathrm{K}$ (de Schutter and Taerwe 1995), $\alpha=\alpha_{m}=30 \times 10^{-6} \mathrm{~K}^{-1}$ (Ulm et al. 1999) $, K=14100 \mathrm{MPa}, K_{m}=31800 \mathrm{MPa}, b=0.55$ (Ulm et al. 2004), $\gamma_{c l}=36-0.25\left(T-T_{0}\right)$ $\mathrm{mN} / \mathrm{m}$ (Zuber and Marchand 2004), $\lambda_{m}=1.9 \mathrm{~W} /(\mathrm{mK})$ (deduced from the experimental values of saturated and a dried cement pastes conductivity given by (Kim et al. 2003)).

The evolution of the viscosity of supercooled water with temperature and liquid pressure by numerous empirical relationships. In this study, one of the most recent proposed by (Harris and Woolf 2004) is chosen ( $p_{l}$ in $\mathrm{MPa}$ and $T$ en $\mathrm{K}$ ):

$$
\eta_{l}\left(T, p_{l}\right)=\exp \left(a_{1}+a_{2} p_{l}+a_{3} \frac{p_{l}}{T}+a_{4} p_{l}^{2}+a_{5} \frac{p_{l}^{2}}{T}+a_{6} p_{l}^{3}+\frac{a_{7}}{T}+\frac{a_{8}}{T^{2}}\right)
$$

with $a_{1}=0.411011 \mathrm{mPa} \mathrm{s}, a_{2}=0.00854113 \mathrm{mPa} \mathrm{s} / \mathrm{MPa}, a_{3}=-2.67340 \mathrm{mPa} \mathrm{s} \mathrm{K} / \mathrm{MPa}$, $a_{4}=-1.35750 \mathrm{mPa} \mathrm{s} / \mathrm{MPa}^{2}, a_{5}=0.00525404 \mathrm{mPa} \mathrm{K} / \mathrm{MPa}^{2}, a_{6}=-5.15042 \mathrm{mPa} \mathrm{s} / \mathrm{MPa}^{3}$, $a_{7}=-2350.32 \mathrm{mPa} \mathrm{s} \mathrm{K}$ and $a_{8}=654401 \mathrm{mPa} \mathrm{s} \mathrm{K}{ }^{2}$.

Let us emphasize that the cement paste did not have air-entraining agent so there was no relief on the hydraulic pressure by the air voids. 


\section{REFERENCES}

Baroghel-Bouny, V., Arnaud, S., Henry, D., Carcasse, M., and Quenard, D. (2002). "Vieillissement des bétons en milieu naturel : une expérimentation pour le $X X I^{e}$ siècle. IIIpropriétés de durabilité des bétons mesurées sur éprouvettes conserveés en laboratoire." Bulletin des Laboratoires des Ponts et Chausseées. 13-59.

Baroghel-Bouny, V., Thierry, M., Barberon, F., Coussy, O., and Villain, G. (2007). "Assessment of transport properties of cementitious materials: a major challenge as regards durability?." Revue Européenne de Génie Civil, accepted.

Bazant, Z., Chern, J., Rosenberg, A., and Gaidis, J. (1988). "Mathematical-model for freezethaw durability of concrete." Journal of the American Ceramic Society, 71, 776-783.

Beaudoin, J. and MacInnis, C. (1974). "The mechanism of frost damage in hardened cement paste." Cement and Concrete Research, 4, 139-147.

Brun, M., Lallemand, A., Quinson, J., and Eyraud, C. (1977). "A new method for the simultaneous determination of the size and the shape of pores : The thermoporometry." Thermochimica Acta, 21, 59-88.

Chateau, X. and Dormieux, L. (2002). "Micromechanics of saturated and unsaturated porous media." International Journal for Numerical and Analytical Methods in Geomechanics, 26, $831-844$.

Coussy, O. (2004). Poromechanics. John Wiley \& Sons.

Coussy, O. (2005). "Poromechanics of freezing materials." Journal of the Mechanics and Physics of Solids, 53, 1689-1718.

Coussy, O. and Fen-Chong, T. (2005). "Crystallization, pore relaxation and microcryosuction in cohesive porous materials." Comptes Rendus Mecanique, 333, 507-512.

Coussy, O. and Monteiro, P. (2007). "Unsaturated poroelasticity for crystallization in pores." Computers and Geotechnics, 34, 279-290.

Cui, L. and Cahyadi, J. (2001). "Permeability and pore structure of OPC cement paste." Cement and Concrete Research, 31, 277-282. 
de Schutter, G. and Taerwe, L. (1995). "Specific head and thermal diffusivity of hardening concrete." Magazine of Concrete Research, 47(172), 203-207.

Dormieux, L. and Bourgeois, E. (2003). Introduction la micromécanique des milieux poreux. Presses de l'Ecole Nationale des Ponts et Chaussées.

Eymard, R., Gallouet, T., and Herbin, R. (2000). The finite volume method.

Fabbri, A. (2006). "Physico-mécanique des matériaux cimentaires soumis au gel-dégel," PhD thesis, Université de Marne-la-Vallée.

Fabbri, A., Fen-Chong, T., and Coussy, O. (2006). "Dielectric capacity, liquid water content, and pore structure of thawing-freezing materials." Cold Region Science and Technology, $44,52-66$.

Fen-Chong, T., Fabbri, A., Guilbaud, J.-P., and Coussy, O. (2004). "Determination of liquid water content and dielectric constant in porous media by the capacitive method." Comptes Rendus Mecanique, 332, 639-645.

Garboczi, E. and Bentz, D. (1996). "Modelling the microstructure and transport properties of concrete." Construction and Building Materials, 10(5), 293-300.

Harris, K. and Woolf, L. (2004). "Temperature and volume dependence of the viscosity of water and heavy water at low temperatures." Journal of Chemical and Engineering Data, 49(4), 1064-1069.

Hearn, N. and Morley, C. (1997). "Self-sealing property of concrete - experimental evidence." Material and structure - Matriaux et constructions, 30, 404-411.

Hervé, E. (2002). "Thermal and thermoelastic behaviour of multiply coated inclusionreinforced composites." International Journal of Solids and Structures, 39, 1041-1058.

Jehng, J., Sprague, D., and Halperin, W. (1996). "Pore structure of hydrating cement paste by magnetic resonance relaxation analysing and freezing." Magnetic Resonance Imaging, 14(7/8), 785-791.

Katz, A. and Thompson, A. (1986). "Quantitative prediction of permeability in porous rock." Physical Review B, 34(11), 8179-8181. 
Kaufmann, J. (1999). "Experimental identification of damage mechanisms in cementitious porous materials on phase transition of pore solution under frost deicing salt attack," $\mathrm{PhD}$ thesis, École Polytechnique Fédérale de Lausanne.

Kim, K.-H., Jeon, S.-E., Kim, J.-K., and Yang, S. (2003). "An experimental study on thermal conductivity of concrete." Cement and Concrete Research, 33, 363-371.

Kleinberg, R. L. and Griffin, D. D. (2005). "NMR measurements of permafrost: unfrozen water assay, pore-scale distribution of ice, and hydraulic permeability of sediments." Cold Regions Science and Technology, 42, 63-77.

LCPC (Décembre 2003). "Recommandations pour la durabilité des bétons durcis soumis au gel." Techniques et méthodes des laboratoires des ponts et chaussées, Laboratoire Central des Ponts et Chaussées".

D. R. Lide, ed. (2001). Handbook of Chemistry and Physics 2001-2002. CRC Press, 82 ${ }^{\text {nd }}$ edition.

Penttala, V. (2006). "Surface and internal deterioration due to saline and non-saline freezethaw loads." Cement and Concrete Research, 36, 921-928.

Pigeon, M. (1984). "Microstructure et résistance au gel des ciments et bétons," PhD thesis, Université Pierre et Marie Curie.

Pigeon, M., Marchand, J., and Pleau, R. (1996). "Frost resistant concrete." Construction and Building Materials, 10(5), 339-348.

Powers, T. (1949). "The air requirement of frost-resistant concrete." Highway Research Board, 29, 184-211.

Powers, T. (1959). "Capillary continuity or discontinuity in cement pastes." PCA Bulletin, $10,2-12$.

Powers, T. and Helmuth, R. (1953). "Theory of volume changes in hardened portland-cement paste during freezing." Highway Research Board, 32, 285-297.

Scherer, G. (1993). "Freezing gels." Journal of Non Crystalline Solids, 155, 1-25.

Sellevold, E. and Bager, D. (1980). "Low temperature calorimetry as a pore structure probe." 
$7^{\text {TH }}$ International Congress on the Chemistry of Cement - Paris, 4, 394-399.

Sellevold, E. and Farstad, T. (1991). "Frost/salt-testing of concrete: Effect of test parameters and concrete moisture history." Nordic Concrete Research, 10.

Shafiq, N. and Cabrera, J. (2004). "Effects of initial curing condition on the fluid transport properties in OPC and fly ash bended cement concrete." Cement $\mathscr{E}$ Concrete Composite, $26,381-387$.

Speedy, R. (1987). "Thermodynamic properties of supercooled water at 1 atm." Journal of Physical Chemistry, 91, 3354-3358.

Ulm, F.-J., Acker, P., and Lévy, M. (1999). "The "chunnel" fire. ii: Analysis of concrete damage." Journal of Engineering Mechanics, 283-289.

Ulm, F.-J., Constantinides, G., and Heukamp, F. (2004). "Is concrete a poromechanics material ? - a multiscale investigation of poroelastic properties." Material and structures/ Concrete Science Engineering, 37, 43-58.

Valenza, J. and Scherer, G. (2005). "Mechanisms of salt scaling." Materials and Structures, $38,479-488$.

Van Genuchten, M. (1980). "A closed form equation for predicting the hydraulic conductivity of unsaturated soils." Soil Science Society of America Journal, 44(5), 892-898.

Verbeck, G. and Klieger, P. (1957). "Studies of salt scaling of concrete." Highway research board bulletin $n^{o} 100$.

Vignes, M. and Dijkema, K. (1974). "A model for the freezing of water in a dispersed medium." Journal of Colloid and Interface Sciences, 49, 165.

Vignes-Adler, M. (1976). "On the origin of the water aspiration in a freezing dispersed medium." Journal of Colloid and Interface Sciences, 60, 162-171.

Zuber, B. and Marchand, J. (2004). "Predicting the volume instability of hydrated cement systems upon freezing using poro-mechanics and local phase equilibra." Concrete Science Engineering, 37(268), 257-270. 


\section{List of Tables}

1 Experimental results of scaling tests . . . . . . . . . . . . . . . . 26

2 Hardened cement pastes characteristics. $S_{c, x^{\circ} \mathrm{C}}$ stands for the ice saturation

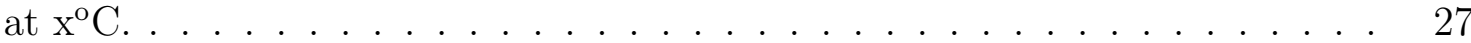




\begin{tabular}{|l||l|l|l|l|}
\hline Scales mass $\left[\mathrm{g} / \mathbf{m}^{2}\right]$ & P4-E-1 & P4-E-2 & P4-E-3d & P4-E-4d \\
\hline \hline 14 cycles & 4 & 27 & Crumbled & Crumbled \\
\hline 28 cycles & 117 & 145 & $*$ & $*$ \\
\hline 42 cycles & 152 & 154 & $*$ & $*$ \\
\hline 56 cycles & 168 & 182 & $*$ & $*$ \\
\hline
\end{tabular}

TABLE 1. Experimental results of scaling tests 


\begin{tabular}{|l||l|l|l|l|}
\hline & $\kappa_{0} \times 10^{20}\left[\mathrm{~m}^{2}\right]$ & $\phi_{0}[\%]$ & $S_{c,-10^{\circ} \mathrm{C}}[\%]$ & $S_{c,-20^{\circ} \mathrm{C}}[\%]$ \\
\hline Virgin & 430 & 28 & 4 & 10 \\
\hline Pre-dried & 4.3 & 29 & 46 & 52 \\
\hline
\end{tabular}

TABLE 2. Hardened cement pastes characteristics. $S_{c, x^{\circ} \mathrm{C}}$ stands for the ice saturation at $\mathrm{x}^{\circ} \mathrm{C}$. 


\section{List of Figures}

1 Freezing-thawing cycle imposed at the sample top surface. . . . . . . . . . . 29

2 Picture of the experimental device. . . . . . . . . . . . . . . . 30

3 Picture of the P4-E-3d cement paste after the first 14 cycles. . . . . . . . . . 31

4 Pictures of the collected scales at different times for the P4-E-1 cement paste. 32

5 Comparison between experimental datas values and fitted $S_{l}(\theta)$ curves for P4-E-1\&2 samples. . . . . . . . . . . . . . . . . . 33

6 Comparison between experimental datas values and fitted $S_{l}(\theta)$ curves for P4-E-3d\& 4d pre-dried samples. . . . . . . . . . . . . . . . . . . 34

7 Liquid and ice pressure profiles for of the virgin and the predried samples at $t=2$ hours. The solid line correspond to the $p_{c}(0, t)=0$ boundary condition while the dot line is for the $\omega(0, t) \cdot n_{0}=0$ one. . . . . . . . . . .

$8 \sigma_{m, x x}$ profiles for of the virgin and the predried samples at $t=2$ hours. The solid line correspond to the $p_{c}(0, t)=0$ boundary condition while the dot line is for the $\omega(0, t) \cdot n_{0}=0$ one. . . . . . . . . . . . . . 36

9 Calculation of the evolution of $\max \left(\sigma_{m, x x}\right)$ for virgin samples. . . . . . . . 37 


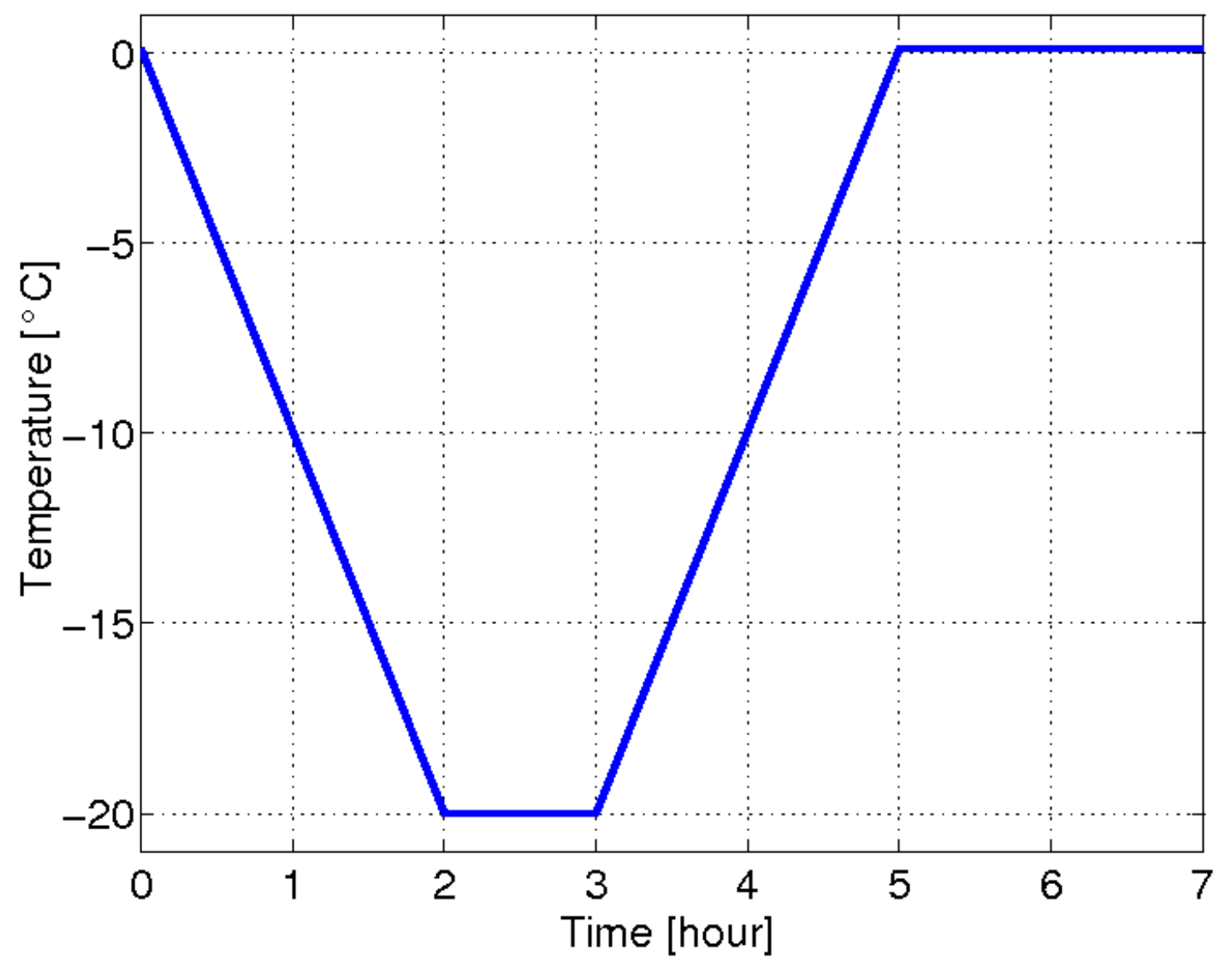

FIG. 1. Freezing-thawing cycle imposed at the sample top surface. 


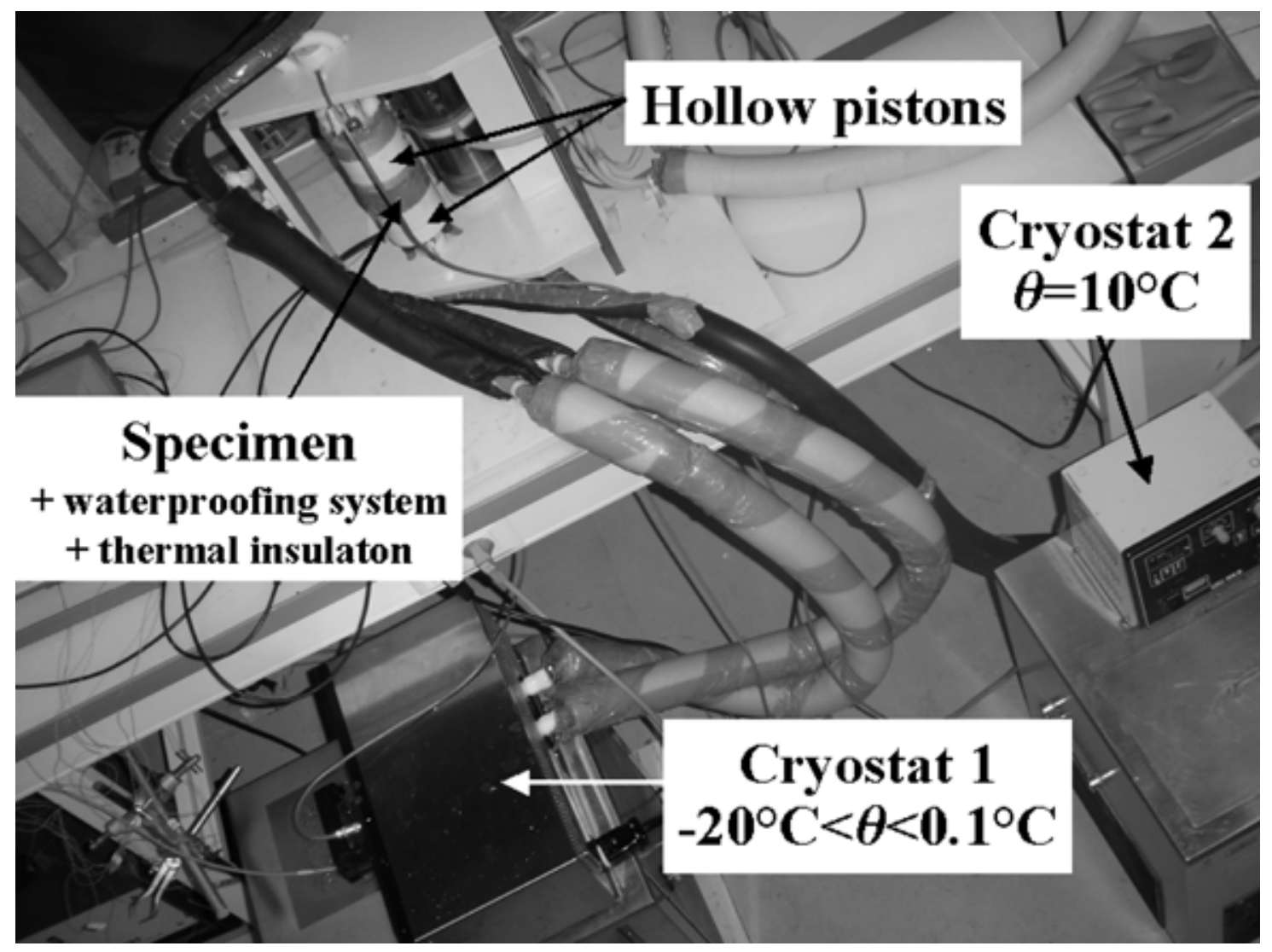

FIG. 2. Picture of the experimental device. 


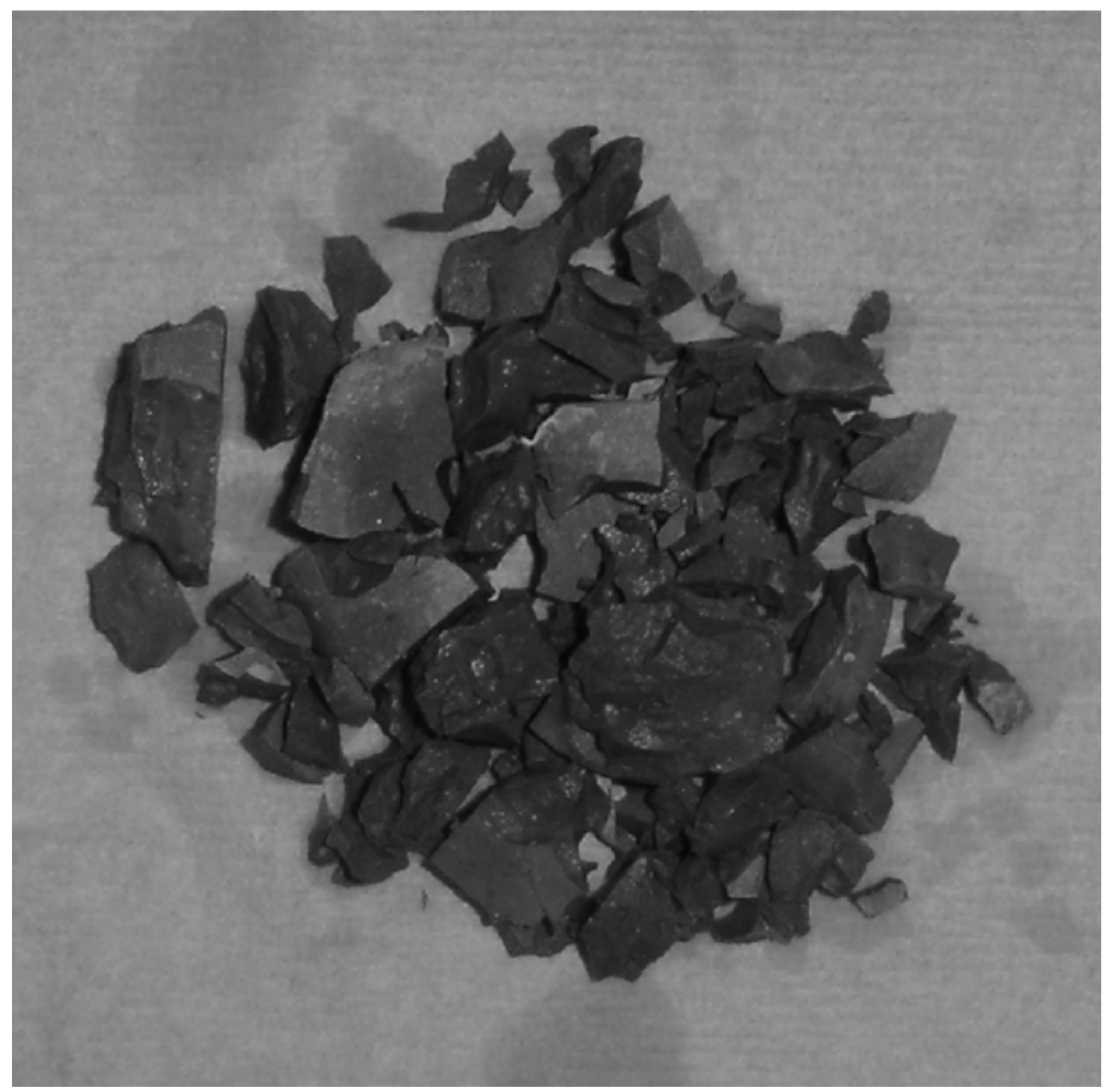

FIG. 3. Picture of the P4-E-3d cement paste after the first 14 cycles. 

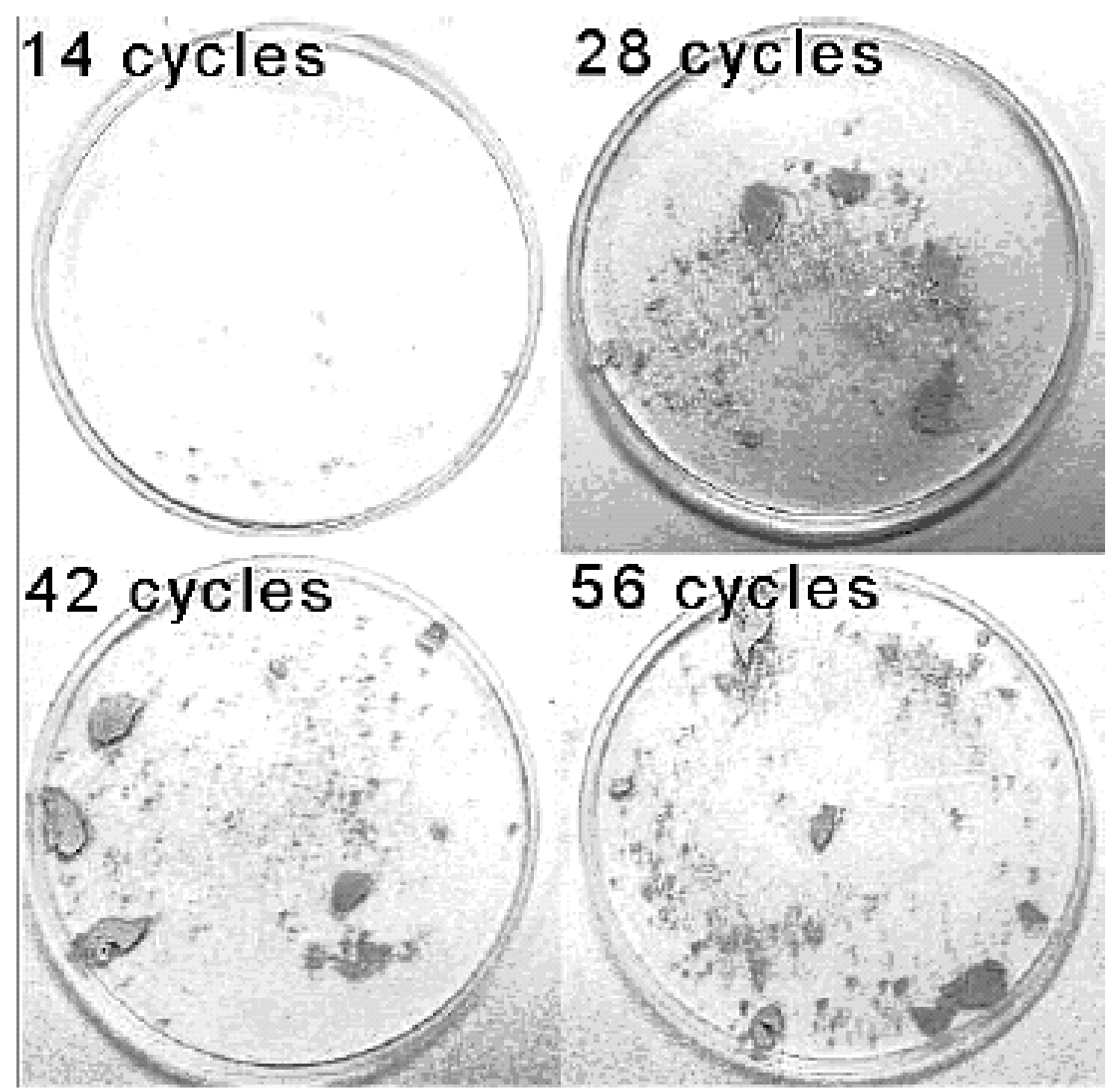

FIG. 4. Pictures of the collected scales at different times for the P4-E-1 cement paste. 


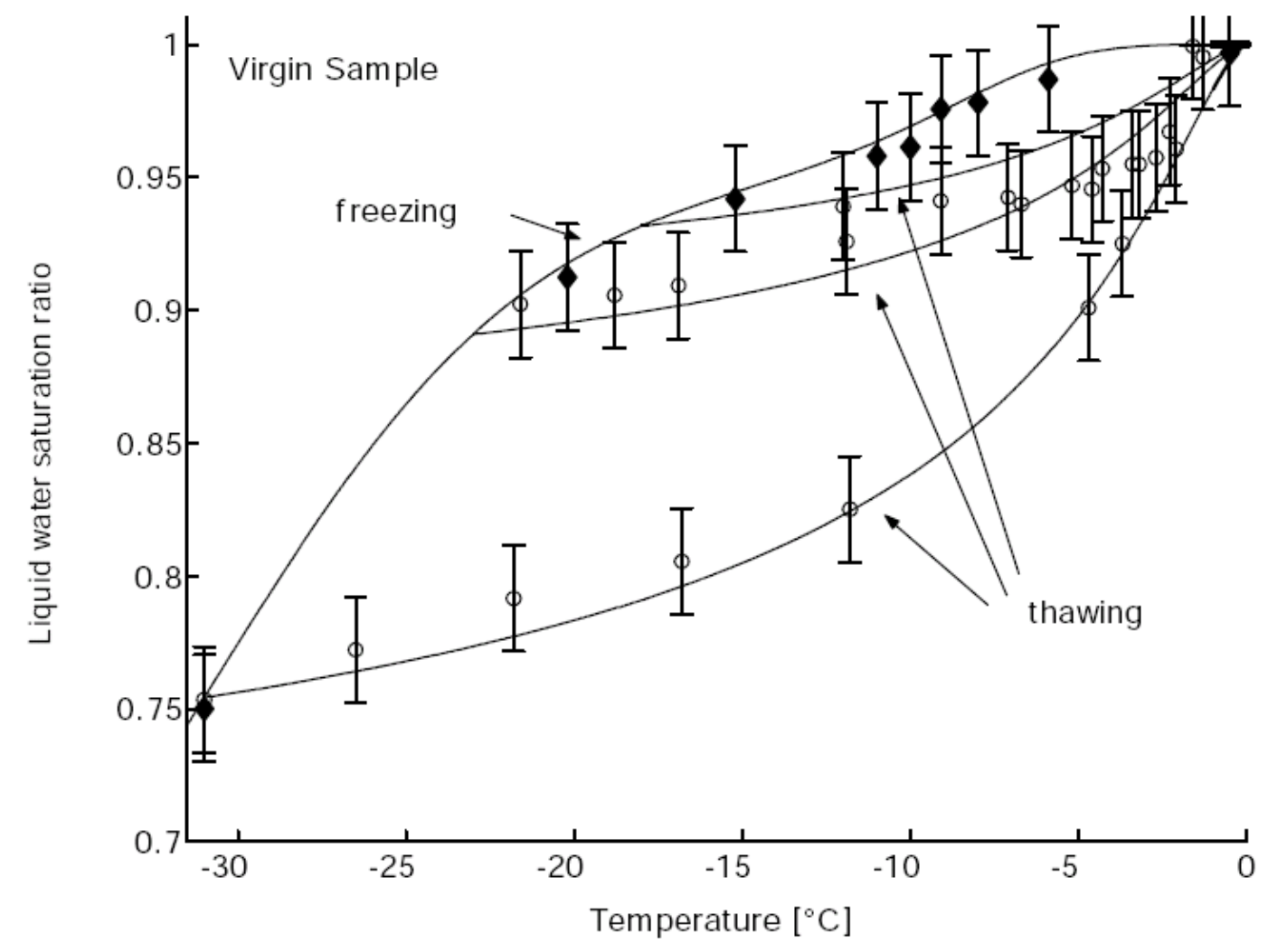

FIG. 5. Comparison between experimental datas values and fitted $S_{l}(\theta)$ curves for P4-E-1\&2 samples. 


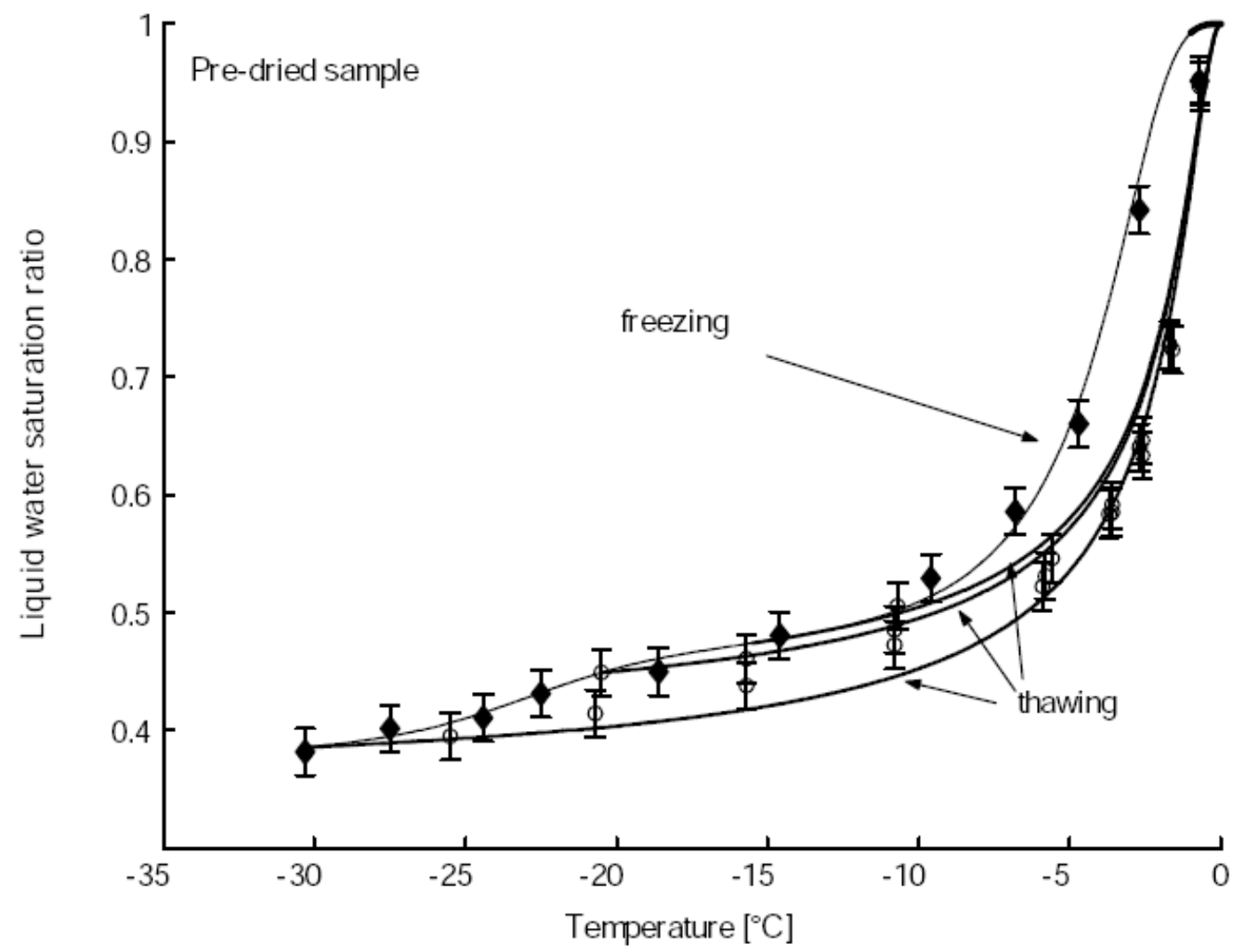

FIG. 6. Comparison between experimental datas values and fitted $S_{l}(\theta)$ curves for P4-E-3d\& 4d pre-dried samples. 

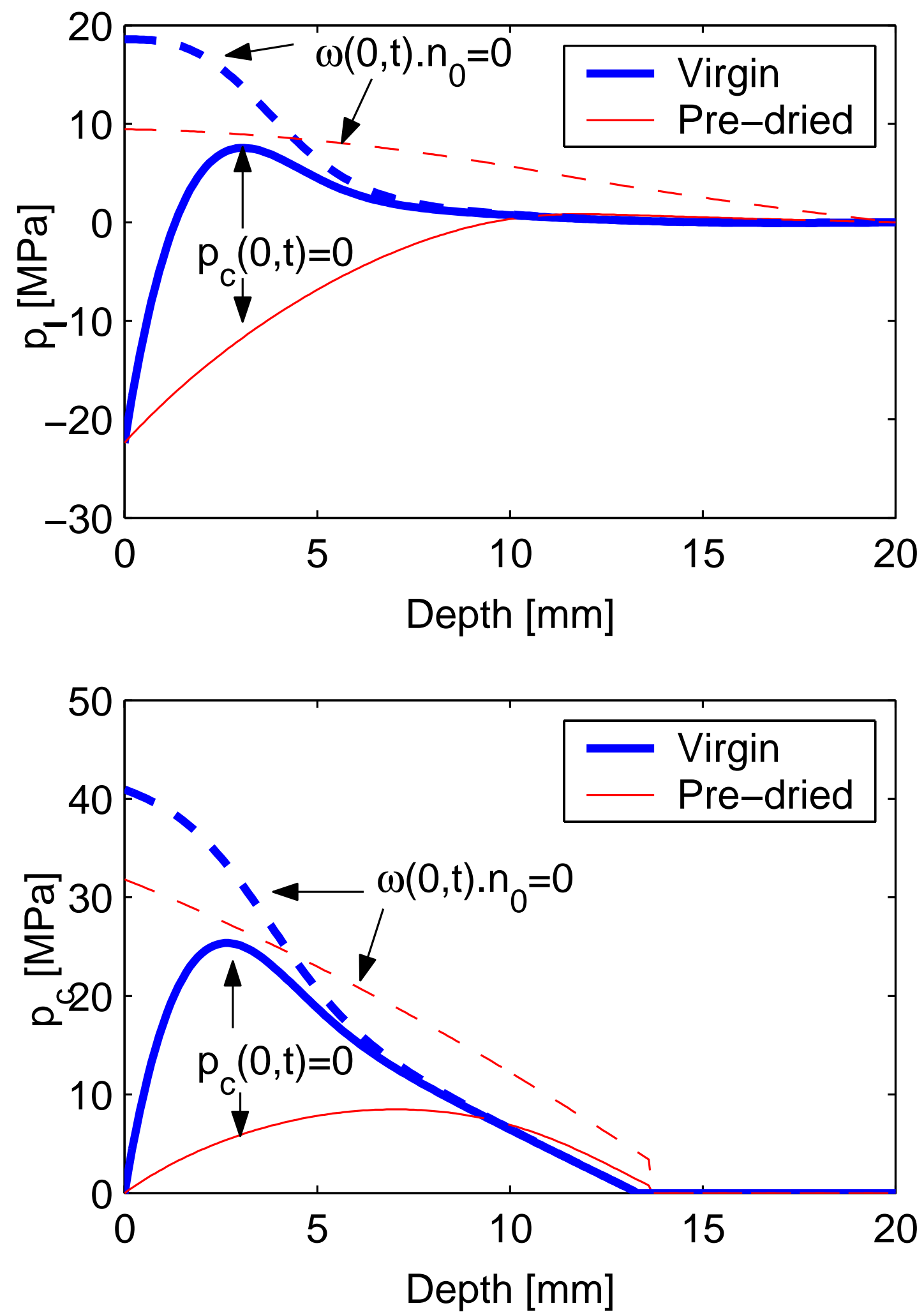

FIG. 7. Liquid and ice pressure profiles for of the virgin and the predried samples at $t=2$ hours. The solid line correspond to the $p_{c}(0, t)=0$ boundary condition while the dot line is for the $\omega(0, t) \cdot n_{0}=0$ one. 


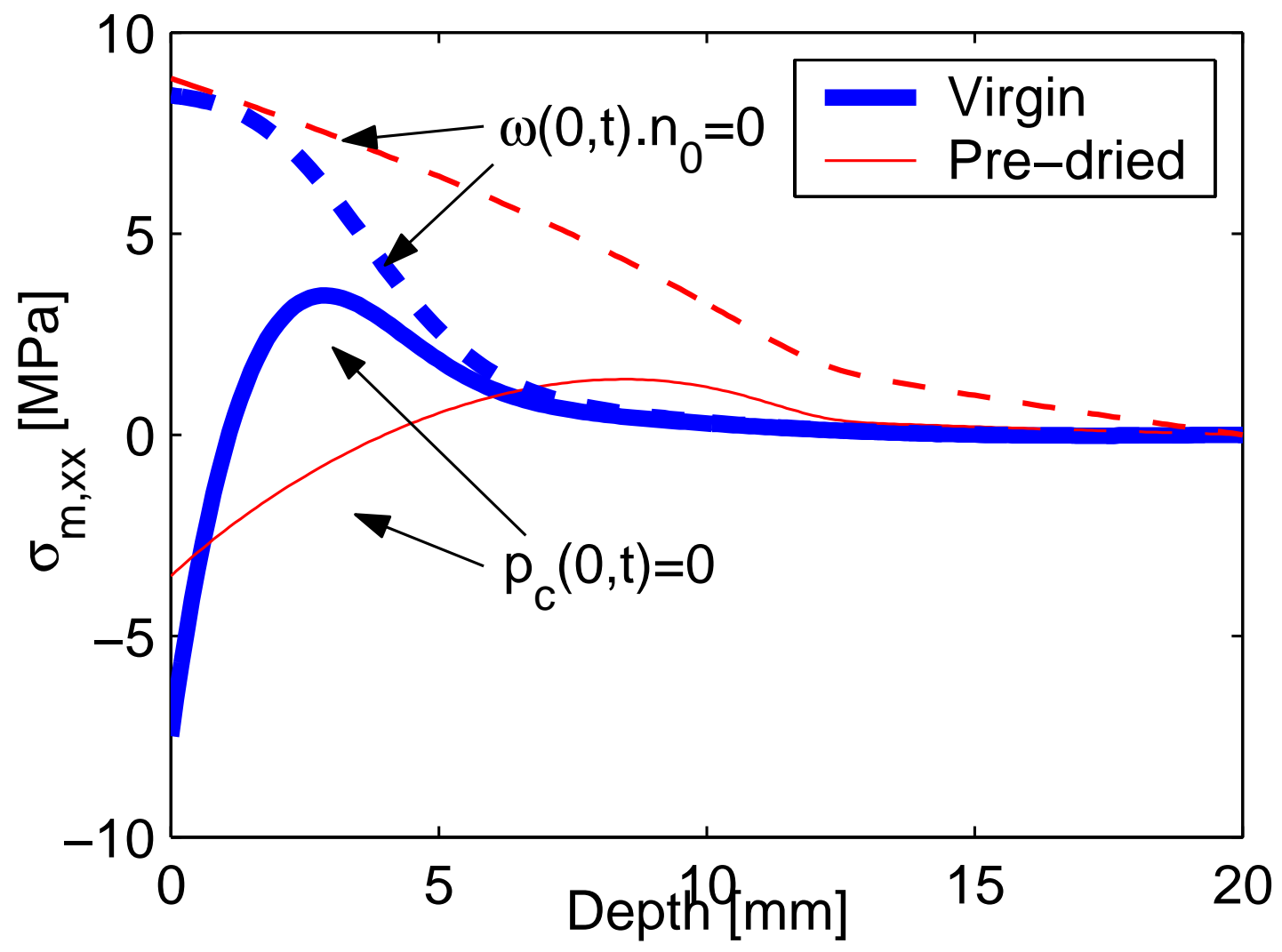

FIG. 8. $\sigma_{m, x x}$ profiles for of the virgin and the predried samples at $t=2$ hours. The solid line correspond to the $p_{c}(0, t)=0$ boundary condition while the dot line is for the $\omega(0, t) \cdot n_{0}=0$ one. 


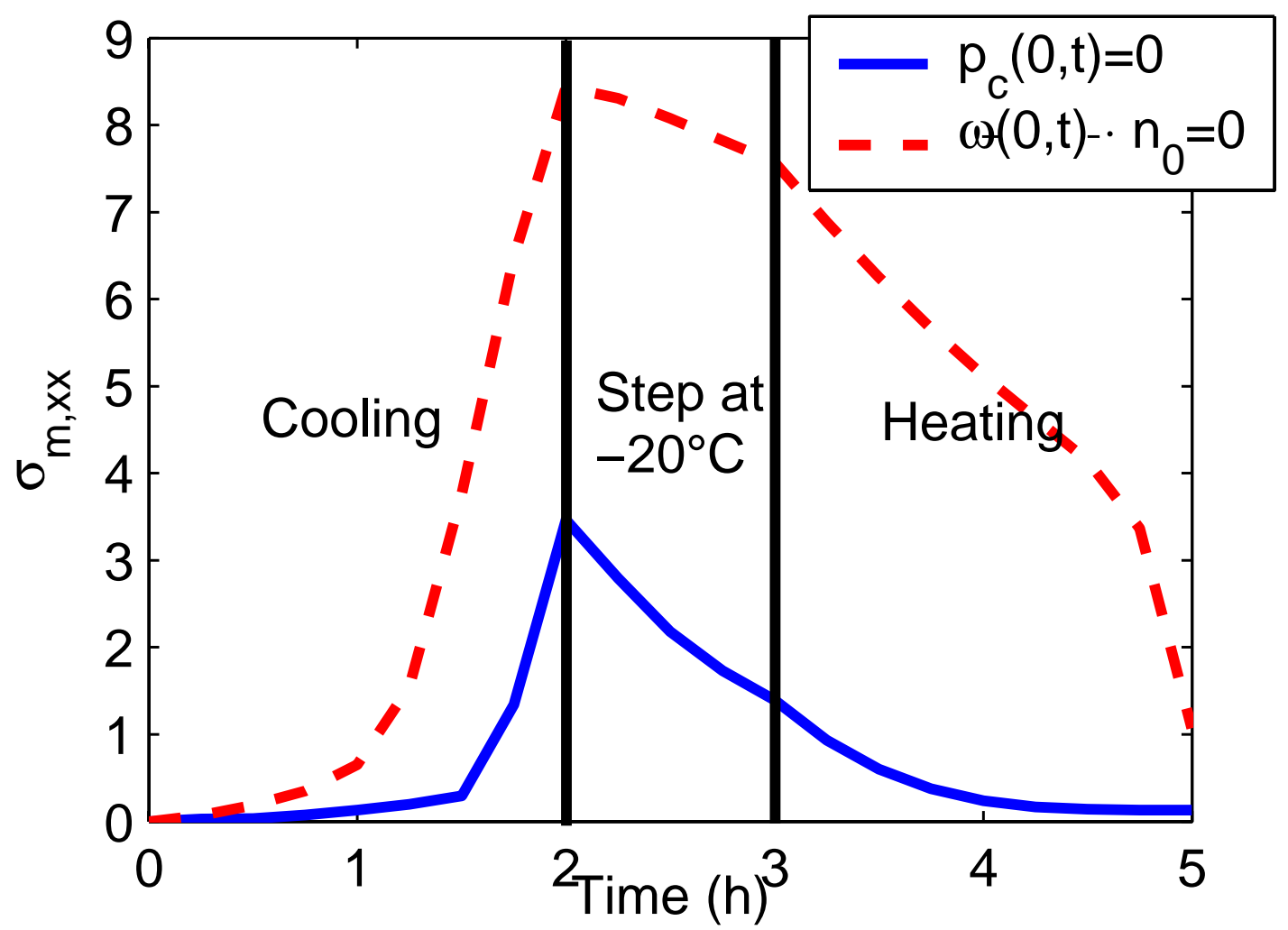

FIG. 9. Calculation of the evolution of $\max \left(\sigma_{m, x x}\right)$ for virgin samples. 